\author{
PII: S0749-6419(98)00027-8
}

\title{
A MODIFIED SWIFT LAW FOR PRESTRAINED MATERIALS
}

\author{
J. V. Fernandes, ${ }^{1 *}$ D. M. Rodrigues, ${ }^{1}$ L. F. Menezes ${ }^{1}$ and M. F. Vieira ${ }^{2}$ \\ ${ }^{1}$ Mechanical Engineering Department (CEMUC), University of Coimbra, Pinhal de Marrocus, \\ 3030 Coimbra, Portugal \\ ${ }^{2}$ Metallurgical Engineering Department (GMM-IMAT), University of Oporto, 4099 Porto Codex, Portugal
}

(Received in final revised form 7 February 1998)

\begin{abstract}
A modified Swift law to describe the evolution of the mechanical behaviour in reloading of prestrained materials is proposed in this work. This equation is deduced from the original Swift law by including a parameter that accounts for the effect of strain path change. This parameter depends on the value of the yield stress and the subsequent work-hardening behaviour in reloading. The new equation predicts well the general mechanical behaviour in the second path for copper and steel. In particular, it predicts accurately the strain value for which necking occurs during reloading and fits experimental stress-strain curves well. The flow equation formulated remains sufficiently simple to be applied in finite element modelling of prestrained materials. However, since the parameter, which is needed for the modified Swift law, must be previously known, the strain path change itself cannot be part of the simulation. (C) 1998 Elsevier Science Ltd. All rights reserved.
\end{abstract}

Key words: mechanical modelling.

\section{INTRODUCTION}

The resolution of problems in plasticity needs equations that describe the strain dependence of the flow stress. To model this dependence, several empirical formulations were suggested. In cold working processes, in which strain hardening is prevalent, the mechanical behaviour is usually described by parabolic equations such as those suggested by Hollomon (1945):

$$
\sigma=k \varepsilon^{n}
$$

or Swift (19.52):

$$
\sigma=k\left(\varepsilon_{0}+\varepsilon\right)^{n}
$$

where $\sigma$ and $\varepsilon$ represent the equivalent stress and strain, respectively, and $k, n$ and $\varepsilon_{0}$ are constants for a particular material, determined usually in uniaxial tension tests.

Satisfactory correlations between theory and experimental data were found for both equations, when carefully determining the adjustable parameters $\left(k, n\right.$ and $\left.\varepsilon_{0}\right)$. However, eqns (1) and (2) are not adequate to model the material behaviour along complex strain

*Corresponding author. 
paths, unless new values for the constants are calculated for the reloading curve. The change of strain path that can occur between successive forming operations, for example, modifies the hardening behaviour. This induces unexpected stress-strain curves during reloading, which cannot be predicted by the equations used for paths without prestrain, such as eqns (1) and (2).

The plastic flow behaviour of metals during sequential strain paths has been a subject of research mainly in steel, aluminium, copper and brass. For a good understanding of this behaviour, several types of two-stage strain path experiments were performed. The more frequent paths used were sequences of uniaxial tension at different angles; plane strain (rolling or plane strain tension)-uniaxial tension or the reverse; equibiaxial stretchinguniaxial tension or the reverse and uniaxial tension-shear. The results concerning the mechanical behaviour in reloading can be described in two contrasting patterns (Ghosh and Backofen, 1973; Wagoner and Laukonis, 1983; Chung and Wagoner, 1986):

- a relatively low initial flow stress (compared with the stress at the same equivalent strain in the monotonic path) is followed by a high work-hardening rate and, when the second path is in tension, the total homogeneous plastic deformation is almost unaffected by the path change, or

- a relatively high initial flow stress is followed by a low work-hardening rate and, depending on the prestrain value, by a reduction in the total homogeneous plastic deformation when the reloading is in tension.

The material used and the type of path change determine the occurrence of these types of behaviours. The first behaviour is typical of Bauschinger experiments, that is, the change in strain path corresponding to an inversion of the loading conditions, such as tension-compression. Also, this behaviour occurs after other strain path changes in the case of brass (Ghosh and Backofen, 1973; Ranta-Eskola, 1980; Doucet and Wagoner, 1989; Wagoner, 1982).

The second behaviour is more general concerning the material and the change of strain path. In recent studies on the plastic deformation of steel (Ranta-Eskola, 1980; Laukonis, 1981; Wagoner and Laukonis, 1983; Schmitt et al., 1985; Raphanel et al., 1986; Doucet and Wagoner, 1989) aluminium (Lloyd and Sang, 1979; Juul Jensen and Hansen, 1990; Wilson et al., 1990) and copper sheets (Vieira et al., 1990; Schmitt et al., 1991; Fernandes et al., 1993; Vieira and Fernandes, 1995), the mechanical response in reloading has been exhaustively analysed. For strain path changes other than inversion of the loading conditions, these metals display a behaviour of the second type. In this case, it is possible to summarise extensive characteristics of the mechanical behaviour during reloading as follows.

1. The yield behaviour in reloading is strongly dependent on the type of strain path change. For a supplied prestrain value, the reloading yield stress is linked to the activation of new slip systems (not active during the prestrain), which depends on the mode of path change. Experimental results show that the value of normalised reloading yield stress in tension (which corresponds to the ratio between the reloading yield stress and the stress during monotonic tension at the same strain) can take values from the unity (corresponding to no change of strain path) to approx 1.15 (for drastic change of strain path, i.e. when in most of the grains the active slip systems during reloading have not been active during the first path). 
2. After a sharp decrease in the work-hardening rate at the beginning of reloading, the hardening curve $\theta(=\mathrm{d} \sigma / \mathrm{d} \varepsilon)=f(\sigma)$ is lower for reloading than along the reference monotonic loading curve and the plastic behaviour tends to the behaviour of the monotonic test.

3. For small prestrains, when the second path is in tension, the total uniform plastic deformation is almost unaffected by the path change. After a critical value of prestrain, the residual uniform strain during the second path in tension depends on the type of the strain path change. For drastic changes of strain path, the localisation of deformation appears sooner than for smooth strain path changes.

4. For a sequence of two strain paths, the first loading mode type hardly affects the mechanical behaviour during the second loading.

The aspects of mechanical behaviour associated with the path change are now well known, but it is still necessary to perform work to model the strain hardening behaviour of metals during complex loading. The present study formulates an equation that describes the mechanical response of prestrained samples at moderate levels. The equation proposed is deduced from the one commonly used in monotonic deformation: the Swift law. The adjustable parameters determined by fitting this equation to the experimental curve without prestrain remain the same in the modified law. This one contains a new parameter that characterises the path change.

\section{GENERAL FORMULATION OF THE MODIFIED SWIFT LAW}

To model the strain hardening of metals subjected to complex strain paths, an equation that considers the principal features of the plastic behaviour is necessary. In their original formulations, the equations proposed for simple tests such as eqns (1) and (2) are inadequate, unless new values for the constants are calculated for the reloading curve as referred to above. An alternative for the hardening equation in reloading is to use a law that can be deduced from those used along monotonic paths by introducing parameters that characterise the strain path change. In this work the choice concerns the Swift equation.

The strain dependence of the flow stress along the second path can be described by the proposed modified Swift equation, as follows

$$
\sigma^{*}=k\left[F\left(\varepsilon_{0}+\varepsilon_{p}\right)+\varepsilon^{*}\right]^{n},
$$

where $k, n$ and $\varepsilon_{0}$ have the same significatice as in eqn (2), $\varepsilon_{p}$ is the equivalent prestrain value, $\sigma^{*}$ and $\varepsilon^{*}$ are the equivalent stress and strain in reloading, respectively, and $F$ is a parameter that depends on the strain path change. This parameter considers the change in stress value associated with the path change and its evolution with deformation.

The parameter $F$ must be a function of $\varepsilon^{*}$ because the stress gap between the reloading curve and the reference curve vanishes with increasing strain. A simple way to describe this dependence is to consider that $F$ is a linear function of $\varepsilon^{*}$

$$
F=a \varepsilon^{*}+b,
$$

where $a$ and $b$ are material constants that depend on the strain path change. So, eqn (3) can be written as 


$$
\sigma^{*}=k\left[g\left(\varepsilon_{0}+\varepsilon_{p}\right)+h \varepsilon^{*}\right]^{n},
$$

where $g$ and $h$ are constants with $g=b$ and $h=\left[a\left(\varepsilon_{0}+\varepsilon_{p}\right)+1\right]$. This equation leads to a simple interpretation of eqn (3): $g$ represents the alteration of the reloading yield stress after the path change ( $g=1$ in its absence) and $h$ characterises the difference in the workhardening behaviour between reloading and the simple strain path $(a=0$, i.e. $h=1$ for the same behaviour).

An important aspect of eqn (5) is that it can have a formulation identical to that of the Swift law as it was originally formulated

$$
\sigma^{*}=k^{*}\left(\varepsilon_{0}^{*}+\varepsilon^{*}\right)^{n}
$$

where $k^{*}=k h^{n}$ and $\varepsilon_{0}^{*}=\frac{g}{h}\left(\varepsilon_{0}+\varepsilon_{p}\right)$.

This new equation must agree with the more significant characteristics of the response in reloading shown in Fig. 1, namely

1. The reloading yield stress $\left(\sigma^{*}\right.$ for $\varepsilon^{*}=0$ ), commonly defined as the back extrapolated stress $\left(\sigma_{b e}\right)$, is higher than the reference stress $\left(\sigma_{r}\right.$ equal to $\sigma$ for $\varepsilon=\varepsilon_{p}$ ) measured at the same equivalent strain on the curve without prestrain, called the reference curve.

2. The work-hardening rate of prestrained metals is lower than that of a virgin sample. This means that the two stress-strain curves meet at a finite strain value $\varepsilon=\alpha n$, where $\alpha$ is a constant close to one (point $B$ in Fig. 1).

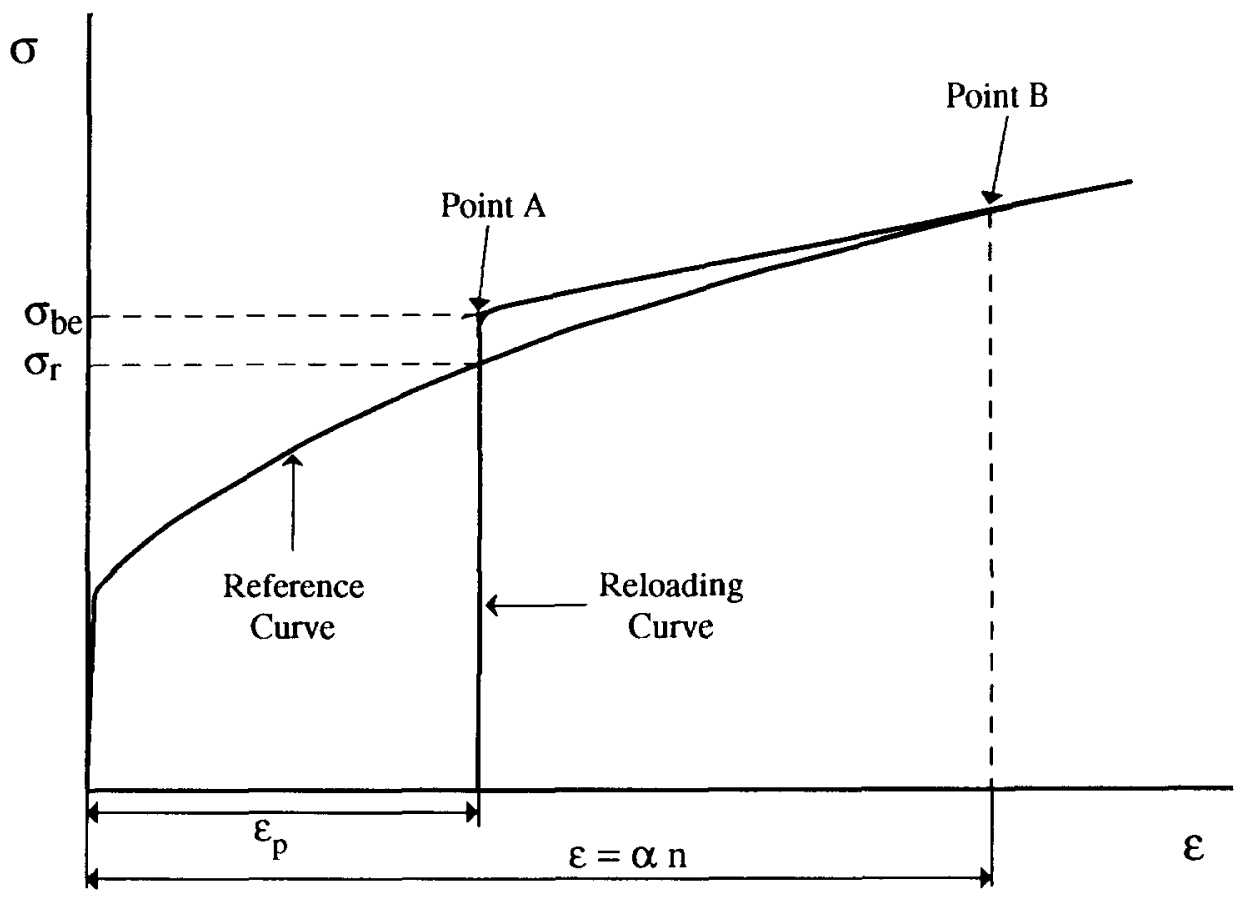

Fig. 1. Schematic representation of true stress-true strain curves in tension without prestrain and after a prestrain to $\varepsilon_{p}$. 
In order to describe fully the strain hardening behaviour of the material in reloading, it is necessary to determine the parameters $g$ and $h$ of eqn (5). This is accomplished by taking into consideration that

1. The stress $\sigma_{A}^{*}$, determined by eqn (6) for $\varepsilon^{*}=0$ (point $A$ in Fig. 1), can be defined as a function of the normalised reloading yield stress $\left(\sigma_{b e} / \sigma_{r}\right)$

$$
\sigma_{A}^{*}=\frac{\sigma_{b e}}{\sigma_{r}} \cdot \sigma_{A},
$$

where $\sigma_{A}$ is the stress determined by eqn (2) at the end of the first path $\left(\varepsilon=\varepsilon_{p}\right)$. In such cases, it is valid to write

$$
\varepsilon^{*}=0 \Rightarrow g=\left(\frac{\sigma_{b e}}{\sigma_{r}}\right)^{\frac{1}{n}} .
$$

2. At the point where the two curves meet (point $B$ in Fig. 1), the stress is the same $\left(\sigma_{B}^{*}=\sigma_{B}\right)$. Therefore, it is possible to write that the strain at point $B\left(\varepsilon_{B}^{*}\right)$ is given by

$$
\varepsilon_{B}^{*}=\alpha n-\varepsilon_{p} \Rightarrow h=\frac{\left(\varepsilon_{0}+\alpha n\right)-\left(\frac{\sigma_{b}}{\sigma_{r}}\right)^{\frac{1}{n}}\left(\varepsilon_{0}+\varepsilon_{p}\right)}{\left(\alpha n-\varepsilon_{p}\right)} .
$$

In this equation, $\alpha$ is a parameter that characterises, after yielding, the influence of the strain path change on the level of the true stress-true strain curve.

Under these assumptions, the modified Swift law for prestrained materials is then expressed by the equation

$$
\sigma^{*}=k\left[\left(\frac{\sigma_{b e}}{\sigma_{r}}\right)^{\frac{1}{n}}\left(\varepsilon_{0}+\varepsilon_{p}\right)+\frac{\left(\varepsilon_{0}+\alpha n\right)-\left(\frac{\sigma_{b}}{\sigma_{r}}\right)^{\frac{1}{n}}\left(\varepsilon_{0}+\varepsilon_{p}\right)}{\left(\alpha n-\varepsilon_{p}\right)} \varepsilon^{*}\right]^{n} .
$$

This equation models the material behaviour on reloading for several complex strain paths. For it to be used, it is necessary to determine the values of the parameters $k, n$ and $\varepsilon_{0}$ for monotonic loading. Moreover, the influence of the strain path history on the value of normalised reloading yield stress $\left(\sigma_{b e} / \sigma_{r}\right)$ must be known. This can be experimentally determined, but it is possible to obtain it from simulations taking into account the developed intragranular dislocation microstructures and the evolution of the deformation textures during the prestrain path (Schmitt et al., 1985; Raphanel et al., 1986). The value of the parameter $\alpha$ in eqn (10) must also be known. This parameter defines the way in which the reloading curve approaches the reference curve and its value depends on the point of coincidence of these curves (point $B$ in Fig. 1). The existence of a single value for the $\alpha$ parameter enables the behaviour to be characterised along different strain paths and in different materials, as will be discussed next. Also, the general applicability of eqn (10) will be considered below, in relation to experimental results concerning the response of copper and steel sheets. 
This law, in particular in the form given by eqn (6), is simple to use in numerical codes where the isotropic work-hardening is described by the Swift law (Menezes et al., 1991). For example, the numerical simulation of tensile tests of prestrain samples can be performed using this law in standard elastoplastic finite element codes, without changes in the codes (Menezes et al., 1997). However, its application is limited to sharp strain path changes. In the case of continuous changes of strain path, one needs to use more complex models as those proposed by Teodosiu and $\mathrm{Hu}$ (1995), which are based on the evolution of adequate sets of internal variables, characterising the microstructural evolution. The model of Teodosiu and $\mathrm{Hu}(1995) \mathrm{can}$ in principal also predict the ratio $\left(\sigma_{b e} / \sigma_{r}\right)$ as a function of a strain path change. As a result, this model can be used for a finite element simulation of a deformation process during which strain path changes take place.

\section{VALIDATION OF THE MODEL}

To evaluate the models for work-hardening behaviour, it is necessary to perform direct comparisons between theoretical and experimental hardening curves. However, the information in a stress-strain curve is confined to each particularly case. So, general information (even if not involving stress directly) is useful to validate the model. The conditions for the appearance of necking, for example, can be used to test models that describe the mechanical behaviour along complex strain paths. In the following, a global evaluation of eqn (10) is accomplished on this basis. Direct comparison with specific experimental stress-strain curves is also performed.

In a previous work (Vieira et al., 1990), it was shown that, in tension, the occurrence of plastic instability in prestrained materials is influenced by the following factores: the monotonic behaviour of the material (through the $n$ value of the reference curve), the prestrain value $\left(\varepsilon_{p}\right)$ and the strain path change (via its influence on the normalised reloading yield stress $\sigma_{b e} / \sigma_{r}$ and on the stress evolution with deformation). In the same work, a phenomenological model was proposed to predict the occurrence of necking during reloading in tension, taking into account the values of these three parameters. According to the model, this plastic instability appears when the reloading yield stress crosses the line that represents a pseudo stress-strain curve. This curve defines the appearance of the neck in $(\sigma, \varepsilon)$ space, according to the equation

$$
\theta(=\mathrm{d} \sigma / \mathrm{d} \varepsilon)=\sigma .
$$

When the monotonic behaviour is simply described using eqn (2), the integration of eqn (11) leads to the following equation for the pseudo curve, taking into consideration that this curve is tangent to the reference curve at the maximum load point, for which $\varepsilon=n-\varepsilon_{0}$ (see schematic illustration in Fig. 2)

$$
\sigma=k \exp \left[n(\ln n-1)+\varepsilon+\varepsilon_{0}\right] .
$$

The value of the normalised reloading yield stress $\left(\sigma_{b e} / \sigma_{r}\right)$ above which the plastic instability occurs immediately after reloading can be estimated from the ratio between eqns (12) and (2) as follows

$$
\frac{\sigma_{b e}}{\sigma_{r}}=\left(\frac{n}{\varepsilon_{p}+\varepsilon_{0}}\right)^{n} \exp \left(\varepsilon_{p}+\varepsilon_{0}-n\right) .
$$




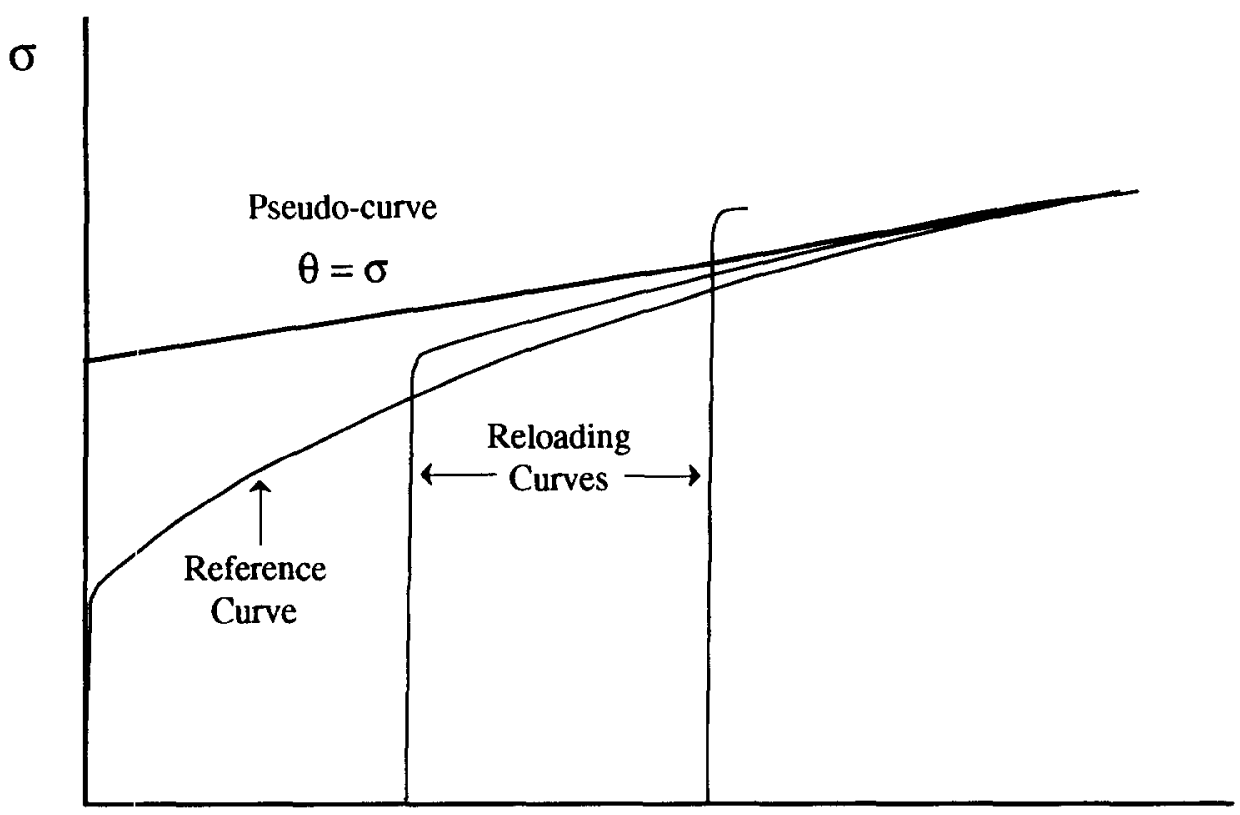

Fig. 2. Schematic representation of the pseudo-curve $\theta=\sigma$ in $(\sigma, \varepsilon)$ space. Also shown are true stress-true strain curves in tension without prestrain and after two different values of prestrain.

From this approach, a limit can be estimated for the normalised reloading yield stress. When the experimental value of this stress is higher than the value obtained from eqn (13), the model predicts that plastic instability appears immediately after reloading. For any experimental value lower than the calculated limit, instability does not occur before the total uniform strain (the prestrain value plus the uniform strain along the second path) approaches the uniform strain value reached in monotonic uniaxial tension.

In this context, an important test of eqn (10) is to check if its results agree with this model concerning the prediction of plastic instability at the beginning of reloading. To do this, it is necessary to solve the equation that defines the beginning of necking $\mathrm{d} \sigma^{*} / \mathrm{d} \varepsilon^{*}=\sigma^{*}$, with a uniform residual strain $\varepsilon_{r}^{*}=\varepsilon^{*}=0$, which gives

$$
\frac{\sigma_{b e}}{\sigma_{r}}=\left(\frac{n}{\varepsilon_{p}+\varepsilon_{0}}\right)^{n}\left(\frac{\varepsilon_{0}+\alpha n}{n+\alpha n-\varepsilon_{p}}\right)^{n} .
$$

From this equation, the evolution of $\sigma_{b e} / \sigma_{r}$ with $\varepsilon_{p}$ can be plotted, for different $\alpha$ values, as shown in Fig. 3. This evolution depends on $\alpha$, and it is possible to choose the value of this parameter that best fits the theoretical evolution predicted by the model, described by eqn (13). The analysis of Fig. 3 allows us to conclude that for copper $(n=0.35)$ and steel ( $n=0.24$ ) the best value for $\alpha$ is close to 0.8 .

The predicted evolution of $\sigma_{b e} / \sigma_{r}$ with $\varepsilon_{p}$ is also compared with experimental results. This is shown in Fig. 4 for copper and low carbon steel sheets. For copper, the strain paths used were sequences of two uniaxial tensions (T-T) at several different angles, 15 , $30,45,60,75$ and $90^{\circ}$ and rolling followed by tension (R-T) with the axis normal and 

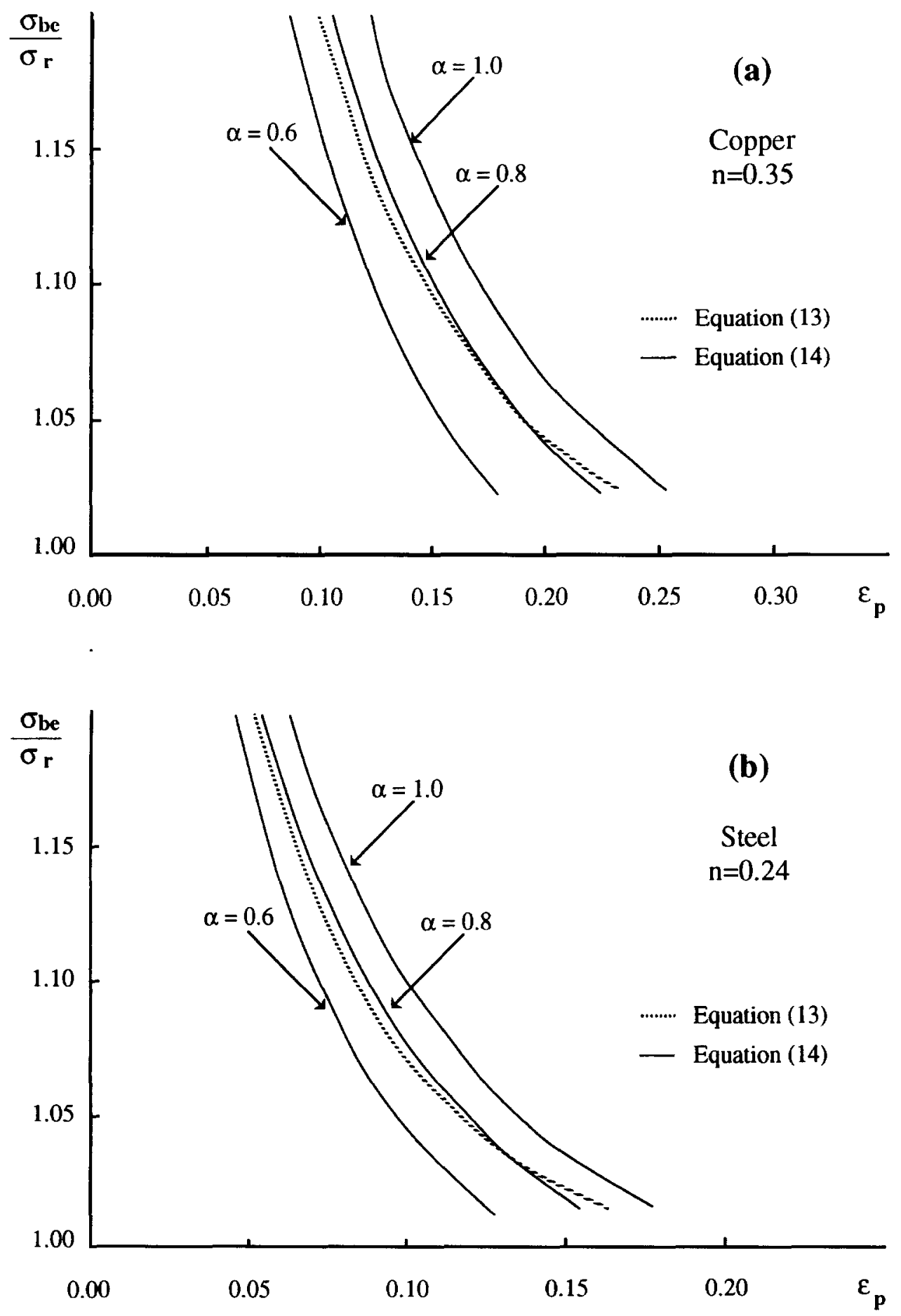

Fig. 3. Evolution of $\sigma_{b e} / \sigma_{r}$ with $\varepsilon_{p}$, according to eqns (13) and (14) for three different $\alpha$ values, for (a) copper and (b) steel. 

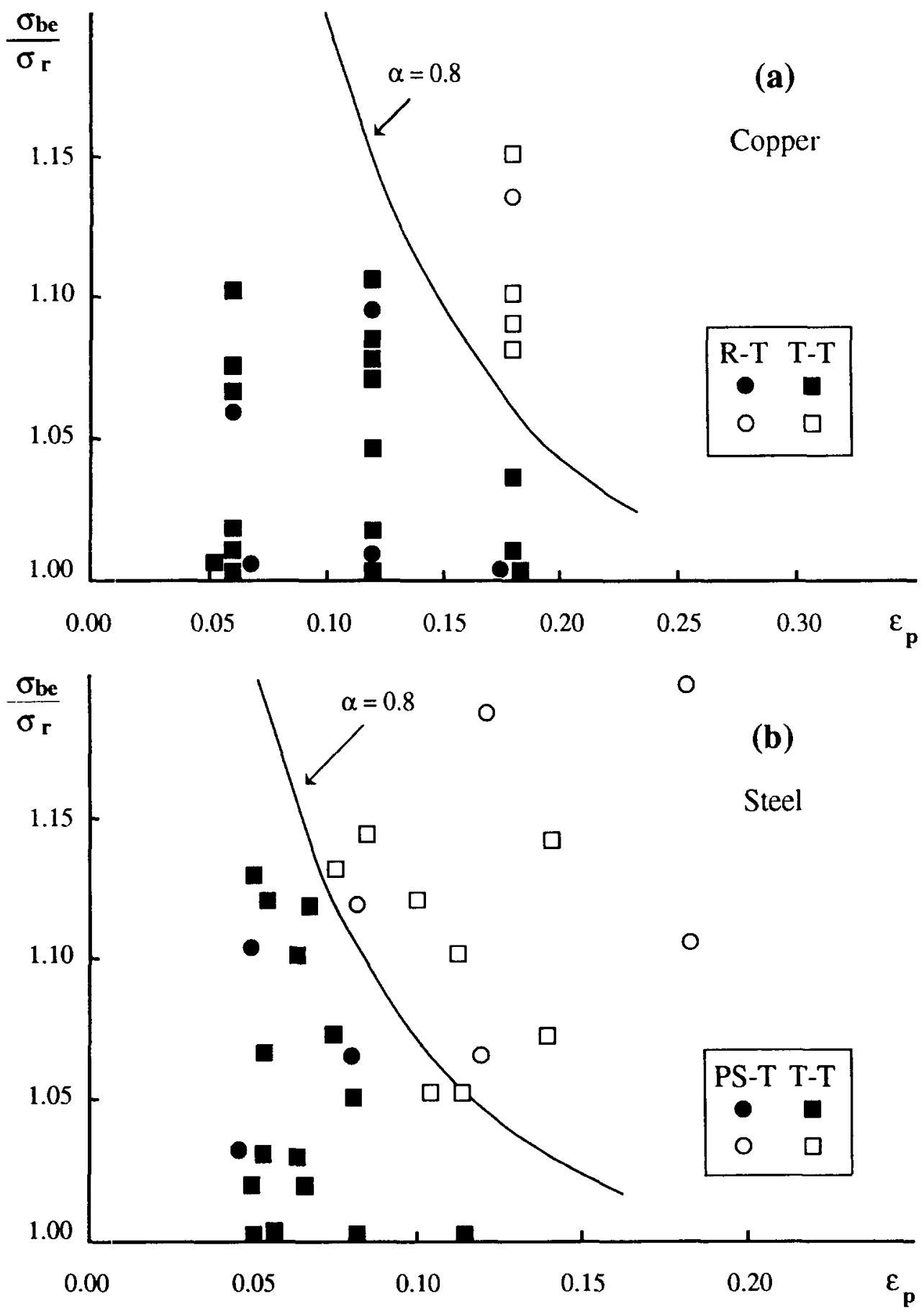

Fig. 4. Evolution of $\sigma_{b e} / \sigma_{r}$ with $\varepsilon_{p}$, according to eqns (13) and (14) with $\alpha=0.8$, for (a) copper and (b) steel. Experimental results for both materials are also shown. Open symbols: necking occurs immediately at the beginning of reloading; filled symbols: the total uniform strain approaches the uniform strain value reached in monotonic uniaxial tension. 
parallel to the rolling direction (Fig. 4(a)) (Vieira et al., 1990). For steel, the experimental results presented in Fig. 4(b) come from other authors (Ranta-Eskola, 1980 and Laukonis, 1981) and concern sequences of uniaxial tension-tension (T-T) at angles of 45 and $90^{\circ}$ and the sequence of plane strain-uniaxial tension (PS-T). This sequence has been performed in two different ways: the maximum principal strains are normal and parallel for both tests. From the analysis of Fig. 4, it can be concluded that the predicted evolution of $\sigma_{b e} / \sigma_{r}$ with $\varepsilon_{p}$ (for $\alpha=0.8$ ) is in agreement with the experimental data. So, for copper and steel, it was possible to choose an $\alpha$ value for the modified Swift law that predicts the appearance of the early plastic instability at the beginning of the second path adequately.

It is also important to test eqn (10) in the case of relatively low prestrain values, for which a non-zero uniform residual strain $\varepsilon_{r}^{*}$ still exists after reloading in tension. In order to achieve this, it is necessary to solve eqn (11) considering that the stress in reloading is given by eqn (10) and that $\varepsilon_{r}^{*}$ can take any value $\varepsilon_{r}^{*}=\varepsilon^{*} \geqslant 0$, as follows

$$
\varepsilon_{r}^{*}=n-\frac{\left(\alpha n-\varepsilon_{p}\right)\left(\varepsilon_{0}+\varepsilon_{p}\right)\left(\frac{\sigma_{b e}}{\sigma_{r}}\right)^{\frac{1}{n}}}{\alpha n+\varepsilon_{0}-\left(\varepsilon_{0}+\varepsilon_{p}\right)\left(\frac{\sigma_{b c}}{\sigma_{r}}\right)^{\frac{1}{n}}} .
$$

From this equation, the evolution of $\varepsilon_{r}$ with $\sigma_{b e} / \sigma_{r}$ can be plotted for copper and steel, as shown in Fig. 5 for $\alpha=0.8$. In Fig. 5(a) is shown the case of copper and three prestrain values, $\varepsilon_{p}=0.06,0.12$ and 0.18 (Vieira et al., 1990). The experimental results also shown concern the prestrain modes mentioned above, as in Fig. 4(a). Figure 5(b) shows the case of steel and concerns the same sequences as in Fig. 4(b) and three prestrain values, $\varepsilon_{p} \approx 0.05,0.08$ and 0.12 (Ranta-Eskola, 1980; Laukonis, 1981). The analysis of these figures allows us to conclude that the model simulates well the experimental behaviour and that the value $\alpha=0.8$ is adequate for this simulation.

Another significant test carried out on eqn (10) concerns the direct comparison with the experimental mechanical behaviour on reloading for the cases in which no plastic instability appears immediately on reloading. Two examples are shown in Fig. 6(a) for copper. This figure presents the reference curve (curve without prestrain) and two experimental true stress-true strain curves obtained in the tension testing of samples prestrained also in uniaxial tension. Here the angle between the two tensile axes was $45^{\circ}$ (Vieira et al., $1990)$ and two prestrain values $\left(\varepsilon_{p}=0.06\right.$ and 0.12$)$ are illustrated. The analytical curves predicted by the modified Swift law are also plotted for the two prestrain values (broken curves). The value of $\sigma_{b e} / \sigma_{r}$ used in eqn (10) is 1.10 and it was obtained from the experimental data. The Swift law parameters $\left(k=461 \mathrm{~N} \mathrm{~mm}^{-2}, n=0.35\right.$ and $\left.\varepsilon_{0}=0.005\right)$ were determined by fitting eqn (2) to the experimental reference curve. Identical procedures were followed for the steel (Fig. 6(b)). This time, the reference curve is presented (with the following Swift law parameters: $k=508 \mathrm{~N} \mathrm{~mm}^{-2}, n=0.24$ and $\varepsilon_{0}=0.002$ ) as well as two experimental true stress-true strain curves obtained in the tension testing of samples prestrained to $\varepsilon_{p} \approx 0.05$ in uniaxial tension. Here the angles between the two tensile axes were $30^{\circ}\left(\sigma_{b e} / \sigma_{r}=1.11\right)$ and $45^{\circ}\left(\sigma_{b e} / \sigma_{r}=1.15\right)$ (Raphanel $e t$ al., 1986; Schmitt, 1986). It can be observed that the experimental and analytical curves are very close for both materials.

In conclusion, the results concerning the mechanical response of polycrystalline metals subject to strain path changes indicate that the modified Swift law proposed in this work describes the mechanical behaviour on reloading, quite adequately. 

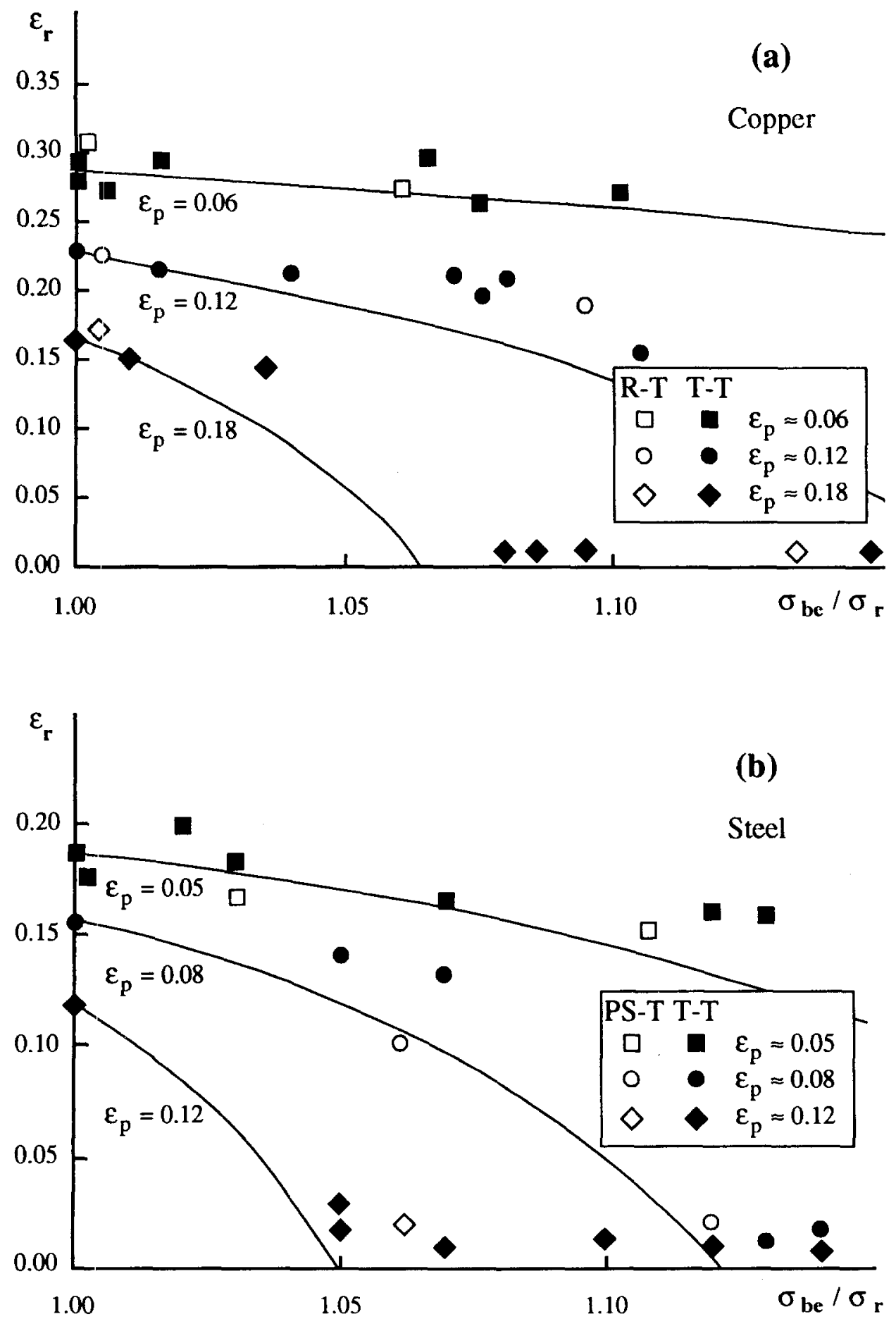

Fig. 5. Dependence of the uniform residual strain $\varepsilon_{r}$ on $\sigma_{b e} / \sigma_{r}$ for (a) copper and (b) steel illustrating the predictions of eqn (15) for three different prestrain values. Experimental results are also shown. 

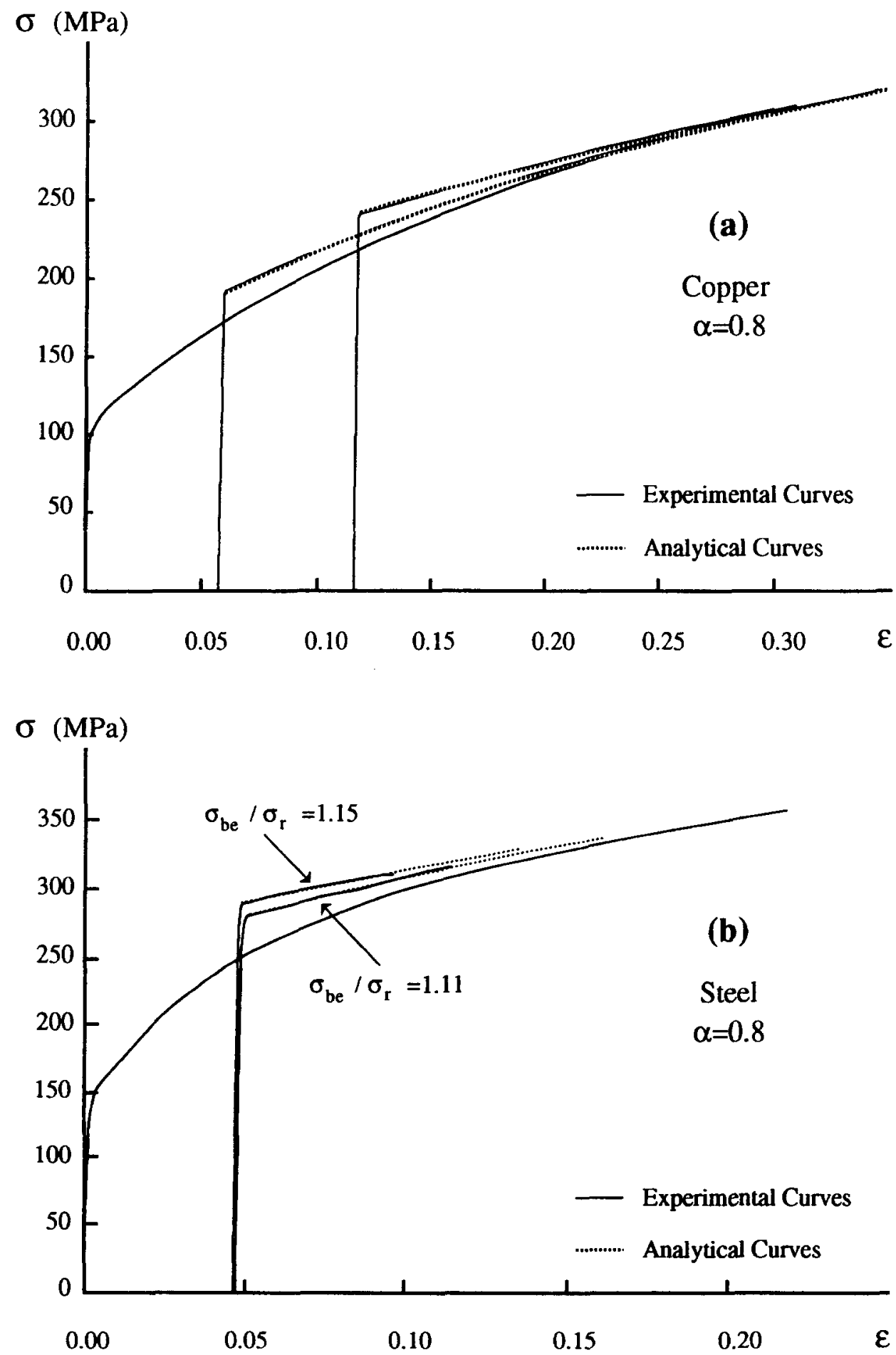

Fig. 6. Experimental and analytical (from eqn (10)) true stress-true strain curves for (a) copper and (b) steel. 


\section{CONCLUSIONS}

In this work, an equation has been formulated to model the flow stress behaviour of metals during reloading. The model employs the Swift law, which is modified to take into account the influence of the strain path change. The alteration of the Swift law concerns the incorporation of a parameter that depends on the change in strain path. In the modified version, the adjustable parameters determined by fitting the original equation to the experimental curve without prestrain remain the same. The new formulation is of the same type as the original Swift law, which simplifies its use in the finite element modelling of prestrained materials. Validation of the model was carried out by comparison with experimental results in the form of stress-strain curves. Also, the results of the model were compared with experimental results concerning the appearance of necking on reloading and with those from a mechanical model used to predict the plastic instability due to necking at the beginning of reloading.

Acknowledgements-The authors are indebted to JNICT for financial support through FEDER and Programa PRAXIS XXI.

\section{REFERENCES}

Chung, K. and Wagoner, R. H. (1987) Effect of stress-strain-law transients on formability. Metall. Trans. 14A, 1487.

Doucet, A. B. and Wagoner, R. H. (1989) Transient tensile behaviour of interstitial-free steel and $70 / 30$ brass following plane-strain deformation. Metall. Trans. 20A, 1483.

Fernandes, J. V., Grácio, J. J. and Schmitt, J.-H, (1993), Grain size effect on the microstructural evolution of copper deformed in rolling-tension, In Large Plastic Deformations: Fundamental Aspects and Applications to Metal Forming, Proceedings of MECAMET'91, ed. J. L. Raphanel and F. Siderof, pp. 219-228. A.A. Balkema, Rotterdam.

Ghosh, A. K. and Backofen, W. A. (1973) Strain hardening and instability in biaxially streched sheets. Metall. Trans. 4A, 1113.

Hollomon, J. H. (1945) Tensile deformation. Trans. AIME 112, 268.

Juul Jensen, [l. and Hansen, N. (1990) Flow stress anisotropy in aluminium. Acta Metall. Mater. 38, 1369.

Laukonis, J. V. (1981) Anisotropic strain localization in tension prestrained sheet steel. Metall. Trans. 12 A, 467.

Lloyd, D. J. and Sang, H. (1979) The infuence of strain path on subsequent mechanical properties-orthogonal tensile paths. Metall. Trans. 10A, 1767.

Menezes, L. F. Teodosiu, C., Makinouchi, A. (1991) 3-D solid elasto-plastic elements for simulating sheet metal forming processes by the finite element method. Proceedings of the FE-Simulation of 3-D Sheet Metal Forming Processes in Automotive Industry. Verein Deutscher Ingenieure, Dusseldorf, pp. 381-403.

Menezes, L. F., Rodrigues, D. M. and Fernandes, J. V. (1997) Numerical simulation of tensile tests of prestrain samples, to be published.

Ranta-Eskola, A. J. (1980) Effect of loading path on stress-strain relationship of sheet steel and brass. Met. Technol. 18, 45 .

Raphanel, J. L., Schmitt, J.-H. and Baudelet, B. (1986) Effect of a prestrain on the subsequent yielding of a low carbon steel sheets: experiments and simulations. International Journal of Plasticity 2, 371.

Schmitt, J.-H (1986) Contribution à l'étude de la Micro-plasticité des Aciers. Ph.D. thesis, Institut National Polytechnique de Grenoble, Grenoble.

Schmitt, J.-H., Aernoudt, E. and Baudelet, B. (1985) Yield loci for polycrystalline metals without texture. Mater. Sci. Eng. 75, 13.

Schmitt, J.-H., Fernandes, J. V., Grácio, J. J. and Vieira, M. F. (1991) Plastic behaviour of copper sheets during sequential tension tests. Mater. Sci. Eng. A 147, 143.

Swift, H. W. (1952) Plastic instability under plane stress. J. Mech. Phys. Solids 1, 1.

Teodosiu, C. and $\mathrm{Hu}, \mathrm{Z}$. (1995) Evolution of the intragranular microstructure at moderate and large strains: modelling and computational significance. In Simulation of Materials Processing: Theory, Methods and Applications, Proceedings of NUNIFORM'95, ed. Shen \& Dawson, pp. 173-182. A. A. Balkema, Rotterdam.

Vieira, M. F. and Fernandes, J. V. (1995) Plastic behaviour of copper sheets subject to a double strain path change. J. Mater. Proc. Tech. 47, 261. 
Vieira, M. F., Schmitt, J.-H., Grácio, J. J. and Fernandes, J. V. (1990) The effect of strain path change on the mechanical behaviour of copper sheets. J. Mater. Proc. Tech. 24, 313.

Wagoner, R. H. (1982) Plastic behaviour of 70-30 brass sheet. Metall. Trans. 13A, 1491.

Wagoner, R. H. and and Laukonis, J. V. (1983) Plastic behaviour of aluminium-killed steel following planestrain deformation. Metall. Trans. 14A, 1487.

Wilson, D. V., Zandrahimi, M. and Roberts, W. T. (1990) Effects of changes in strain path on work hardening in $\mathrm{CP}$ aluminium and an $\mathrm{Al}-\mathrm{Cu}-\mathrm{Mg}$ alloy. Acta Metall. Mater. 38, 215. 